Commun. Korean Math. Soc. 25 (2010), No. 4, pp. 647-653

DOI 10.4134/CKMS.2010.25.4.647

\title{
RETRIAL QUEUEING SYSTEM WITH COLLISION AND IMPATIENCE
}

\author{
JEONGSIM KIM
}

\begin{abstract}
We consider an $\mathrm{M} / \mathrm{M} / 1$ retrial queue with collision and impatience. It is shown that the generating functions of the joint distributions of the server state and the number of customers in the orbit at steady state can be expressed in terms of the confluent hypergeometric functions. We find the performance characteristics of the system such as the blocking probability and the mean number of customers in the orbit.
\end{abstract}

\section{Introduction}

Retrial queues are queueing systems in which arriving customers who find all servers occupied may retry for service again after a random amount of time. Retrial queues have been widely used to model many problems in telephone systems, call centers, telecommunication networks, computer networks and computer systems, and in daily life. Detailed overviews for retrial queues can be found in the bibliographies [3, 4, 5], the surveys [9, 12], and the books $[6,10]$.

Retrial queues are characterized by the following feature: If the server is idle when a customer arrives from outside the system, this customer begins to be served immediately and leaves the system after service is completed. On the other hand, any customer who finds the server busy upon arrival joins a retrial group, called an orbit. If the server is idle when a customer from the orbit attempts service, this customer receives service immediately and leaves the system after service completion. Otherwise the customer comes back to the orbit immediately and repeats the retrial process.

For example, consider a call center scenario. Typically a call center consists of a finite number of servers that answer customer calls. In a queueing model of a call center, the customers are callers, the servers are telephone agents (operators or communications equipment), and queues are populated by callers

Received August 16, 2009; Revised April 14, 2010.

2000 Mathematics Subject Classification. 60K25.

Key words and phrases. M/M/1 retrial queue, queue length, confluent hypergeometric function.

This work was supported by the research grant of the Chungbuk National University in 2009 
that await service. The operations of the call center can be described as follows: When a customer call arrives, it will be served immediately if a server is available. If all servers are busy with other calls, the customer will be put on hold, and will be asked to wait until a server becomes available. The call center may choose to announce an expected waiting time to the customer at this point. Some customers are patient enough to wait for a server to become available. Other customers will hang up immediately upon hearing the waiting time announcement or abandon after waiting for some time. It is known that in a call center scenario, the impact of customer retrial phenomenon cannot be ignored for the performance of the whole system, see $[1,2]$. Therefore $\mathrm{M} / \mathrm{M} / \mathrm{m}$ retrial queue with impatience arises naturally as a model of a call center. In recent publications $[2,7]$, the authors modelled a call center as the $\mathrm{M} / \mathrm{M} / \mathrm{m}$ retrial queue with exponential impatience times. Aguir et al. [2] proposed a fluid approximation to estimate the stationary retrial rate in the system. Artalejo and Pla [7] presented simple approximations based on truncation for obtaining the number of customers in the system.

Due to the complexity of the analysis in the $\mathrm{M} / \mathrm{M} / \mathrm{m}$ retrial queue with impatience, no explicit formulas are obtained for any probability characteristics of retrial queues with multiple servers. Hence, to obtain an exact analytical expression for the steady state probabilities, we consider the $M / M / 1$ retrial queue with impatience, which has the following additional feature: If an arriving customer finds the server busy, then the arriving customer collides with a customer in service, both customers join the orbit and the server becomes idle immediately. For instance, in the unslotted 1- and $p_{i}$-persistent Carrier Sense Multiple Access with Collision Detection (CSMA-CD) protocols for a fiber optic bus network with a finite number of stations, each of which has an infinite storage buffer, the collisions occur during the transmission of arbitrary length packets because no slot synchronization is needed. See, for example, [11] for a single server retrial queue with collisions.

In this paper, we consider the $\mathrm{M} / \mathrm{M} / 1$ retrial queue with collision and impatience. We show that the generating functions of the joint distributions of the server state and the number of customers in the orbit at steady state can be expressed in terms of the confluent hypergeometric functions. In addition, we find the performance characteristics of the system such as the blocking probability and the mean number of customers in the orbit.

\section{Joint distribution of server state and queue length}

Consider a single server retrial queue where customers arrive from outside the system according to a Poisson process with rate $\lambda$. If the server is idle, the arriving customer/the customer from the orbit begins to be served immediately and leaves the system after service is competed. The service times of customers are exponentially distributed with mean $\mu^{-1}$. If the server is busy, the arriving customer/the customer from the orbit collides with the customer in service 
and both result in being shifted to the orbit. The customer in the orbit either attempts service again after a random time or gives up receiving service and leaves the system after a random time. The inter-retrial time and impatience time of each customer in the orbit are exponentially distributed with mean $\nu^{-1}$ and $\delta^{-1}$, respectively. The arrival process, the service times, the impatience times, and the retrial times are assumed to be mutually independent.

Let $N(t)$ denote the number of customers in the orbit at time $t$. Let $X(t)=1$ or 0 according as the server is busy or idle at time $t$. The process $\{(N(t), X(t))$ : $t \geq 0\}$ is a Markov process with state space $\{0,1,2, \ldots\} \times\{0,1\}$. Let

$$
\begin{aligned}
& p_{n}=\lim _{t \rightarrow \infty} \mathbb{P}\{N(t)=n, X(t)=1\}, \\
& q_{n}=\lim _{t \rightarrow \infty} \mathbb{P}\{N(t)=n, X(t)=0\},
\end{aligned}
$$

be the joint distributions of the server state and the queue length (i.e., the number of customers in the orbit) at steady state. The corresponding generating functions are denoted by

$$
p(z)=\sum_{n=0}^{\infty} p_{n} z^{n}, q(z)=\sum_{n=0}^{\infty} q_{n} z^{n} .
$$

In the following theorem, it is shown that the generating functions of the joint distributions can be expressed in terms of the confluent hypergeometric functions (see e.g., [8]), which is defined by

$$
{ }_{1} F_{1}(\alpha, \beta ; x)=\sum_{n=0}^{\infty} \frac{(\alpha)_{n}}{(\beta)_{n}} \frac{x^{n}}{n !}, \beta \neq 0,-1,-2, \ldots,
$$

where

$$
(\tau)_{n}= \begin{cases}1 & \text { for } n=0 \\ \tau(\tau+1) \cdots(\tau+n-1) & \text { for } n \geq 1\end{cases}
$$

is the Pochhammer symbol.

Theorem 1. For the $M / M / 1$ retrial queue with collision and impatience, the joint distributions of the server state and the queue length in the steady state are given by the following generating functions:

$$
\begin{aligned}
p(z)= & g(0) e^{\frac{\lambda}{\delta} z}{ }_{1} F_{1}\left(\frac{C}{A}, B ; A\left(\frac{\delta}{2 \nu+\delta}-z\right)\right) \\
q(z)= & g(0) e^{\frac{\lambda}{\delta} z}\left[\left(z+\frac{\mu}{\lambda}\right){ }_{1} F_{1}\left(\frac{C}{A}, B ; A\left(\frac{\delta}{2 \nu+\delta}-z\right)\right)\right. \\
& \left.+\frac{C \delta}{B \lambda(\nu+\delta)}(\delta-(2 \nu+\delta) z){ }_{1} F_{1}\left(\frac{C}{A}+1, B+1 ; A\left(\frac{\delta}{2 \nu+\delta}-z\right)\right)\right]
\end{aligned}
$$


where

$$
\left\{\begin{array}{l}
A=\frac{2 \lambda(\nu+\delta)}{\delta(2 \nu+\delta)} \\
B=\frac{\delta^{3}+4 \nu \delta^{2}+2 \lambda \delta^{2}+\mu \delta^{2}+4 \nu^{2} \delta+3 \nu \mu \delta+2 \lambda \nu \delta+2 \nu^{2} \mu}{\delta(2 \nu+\delta)^{2}} \\
C=\frac{\lambda(\delta+\nu)(\delta+\mu)}{\delta^{2}(2 \nu+\delta)},
\end{array}\right.
$$

and

$$
g(0)=e^{-\frac{\lambda}{\delta}}\left[\left(2+\frac{\mu}{\lambda}\right){ }_{1} F_{1}\left(\frac{C}{A}, B ;-\frac{2 \nu A}{2 \nu+\delta}\right)\right.
$$

$$
\left.-\frac{2 C \nu \delta}{B \lambda(\nu+\delta)}{ }_{1} F_{1}\left(\frac{C}{A}+1, B+1 ;-\frac{2 \nu A}{2 \nu+\delta}\right)\right]^{-1},
$$

with ${ }_{1} F_{1}$ denoting the confluent hypergeometric function given in (1).

Proof. Following the standard procedure, we obtain the equilibrium equations:

$$
\begin{aligned}
& \{\lambda+\mu+n(\nu+\delta)\} p_{n}=\lambda q_{n}+(n+1) \nu q_{n+1}+(n+1) \delta p_{n+1}, \\
& \{\lambda+n(\nu+\delta)\} q_{n}=\lambda p_{n-2}+(n-1) \nu p_{n-1}+(n+1) \delta q_{n+1}+\mu p_{n},
\end{aligned}
$$

with the convention that $p_{-1}=p_{-2}=0$. Multiplying both sides of each equation by $z^{n}$ and summing over all $n$, we obtain

$$
\begin{aligned}
& \{(\nu+\delta) z-\delta\} p^{\prime}(z)-\nu q^{\prime}(z)=-(\lambda+\mu) p(z)+\lambda q(z), \\
& -\nu z^{2} p^{\prime}(z)+\{(\nu+\delta) z-\delta\} q^{\prime}(z)=\left(\lambda z^{2}+\mu\right) p(z)-\lambda q(z) .
\end{aligned}
$$

Eliminating $q^{\prime}(z)$ from (4) and (5), we have

(6) $\delta\{(2 \nu+\delta) z-\delta\} p^{\prime}(z)=\{\nu \lambda z-(\nu \mu+\delta \lambda+\delta \mu)\} p(z)+\lambda(\nu+\delta) q(z)$.

Differentiating (6) with respect to $z$ yields

$$
\begin{aligned}
& \delta\{(2 \nu+\delta) z-\delta\} p^{\prime \prime}(z)=\left[\nu \lambda z-\left\{\delta^{2}+(\lambda+\mu+2 \nu) \delta+\nu \mu\right\}\right] p^{\prime}(z) \\
& +\nu \lambda p(z)+\lambda(\nu+\delta) q^{\prime}(z) \text {. }
\end{aligned}
$$

Eliminating $q^{\prime}(z)$ from (4) and (7), we have

(8)

$$
\begin{aligned}
& \nu \delta\{(2 \nu+\delta) z-\delta\} p^{\prime \prime}(z) \\
= & \left\{\left(2 \nu^{2} \lambda+2 \lambda \nu \delta+\lambda \delta^{2}\right) z-\left(\nu \delta^{2}+2 \nu \delta \lambda+\nu \delta \mu+2 \nu^{2} \delta+\nu^{2} \mu+\lambda \delta^{2}\right)\right\} p^{\prime}(z) \\
& +\left(\lambda^{2} \nu+\lambda \nu \mu+\lambda^{2} \delta+\lambda \delta \mu+\nu^{2} \lambda\right) p(z)-\lambda^{2}(\nu+\delta) q(z) .
\end{aligned}
$$

Eliminating $q(z)$ from (6) and (8), we get the following differential equation for $p(z)$ :

$$
\begin{aligned}
\delta\{(2 \nu+\delta) z-\delta\} p^{\prime \prime}(z)= & \left\{2 \nu \lambda z-\left(\delta^{2}+\delta \mu+2 \delta \lambda+2 \delta \nu+\nu \mu\right)\right\} p^{\prime}(z) \\
& +\lambda\{\lambda z+(\lambda+\nu)\} p(z) .
\end{aligned}
$$

Letting

$$
f(z)=e^{-\frac{\lambda}{\delta} z} p(z)
$$


we can rewrite (9) as

$$
\begin{aligned}
\delta\{(2 \nu+\delta) z-\delta\} f^{\prime \prime}(z)+ & \left\{2 \lambda(\nu+\delta) z+\left(\delta^{2}+\delta \mu+2 \delta \nu+\nu \mu\right)\right\} f^{\prime}(z) \\
+ & \left(\lambda \delta+\lambda \mu+\lambda \nu+\frac{\lambda \nu \mu}{\delta}\right) f(z)=0 .
\end{aligned}
$$

The transformations

$$
x=z-\frac{\delta}{2 \nu+\delta} \text { and } g(x)=f(z)
$$

translate (11) into

$$
x g^{\prime \prime}(x)+(A x+B) g^{\prime}(x)+C g(x)=0,
$$

where $A, B, C$ are given in (2). Solving the above differential equation, we have

$$
g(x)=g(0){ }_{1} F_{1}\left(\frac{C}{A}, B ;-A x\right),
$$

with ${ }_{1} F_{1}$ given in (1). According to (10) and (12), we have $p(z)=e^{\frac{\lambda}{\delta} z} g(z-$ $\left.\frac{\delta}{2 \nu+\delta}\right)$, and so

$$
p(z)=g(0) e^{\frac{\lambda}{\delta} z}{ }_{1} F_{1}\left(\frac{C}{A}, B ; A\left(\frac{\delta}{2 \nu+\delta}-z\right)\right) .
$$

Differentiating (13) with respect to $z$ gives

$$
\begin{aligned}
p^{\prime}(z)=g(0) e^{\frac{\lambda}{\delta} z} & {\left[\frac{\lambda}{\delta}{ }_{1} F_{1}\left(\frac{C}{A}, B ; A\left(\frac{\delta}{2 \nu+\delta}-z\right)\right)\right.} \\
& \left.-\frac{C}{B}{ }_{1} F_{1}\left(\frac{C}{A}+1, B+1 ; A\left(\frac{\delta}{2 \nu+\delta}-z\right)\right)\right],
\end{aligned}
$$

because $\frac{d}{d x}{ }_{1} F_{1}(\alpha, \beta ; \gamma x)=\gamma \frac{\alpha}{\beta}{ }_{1} F_{1}(\alpha+1, \beta+1 ; \gamma x)$. Substituting (13) and (14) into (6) and doing simple manipulations yields

$$
\begin{aligned}
q(z)=g(0) e^{\frac{\lambda}{\delta} z} & {\left[\left(z+\frac{\mu}{\lambda}\right){ }_{1} F_{1}\left(\frac{C}{A}, B ; A\left(\frac{\delta}{2 \nu+\delta}-z\right)\right)\right.} \\
& \left.+(D z+E){ }_{1} F_{1}\left(\frac{C}{A}+1, B+1 ; A\left(\frac{\delta}{2 \nu+\delta}-z\right)\right)\right],
\end{aligned}
$$

where $D=-\frac{\delta(2 \nu+\delta)}{\lambda(\nu+\delta)} \frac{C}{B}$ and $E=\frac{\delta^{2}}{\lambda(\nu+\delta)} \frac{C}{B}$. Finally, the constant $g(0)$ is determined by the normalization condition $p(1)+q(1)=1$, which implies (3).

\section{Performance characteristics}

In this section, with the help of generating functions $p(z), q(z)$ given in Theorem 1, we get performance characteristics of the system such as the blocking probability and the mean number of customers in the orbit. 
Blocking probability. The blocking probability $P_{b}$, i.e., probability that the server is busy, is given by $P_{b}=p(1)$. According to Theorem 1 , we have

$$
P_{b}=g(0) e^{\frac{\lambda}{\delta}}{ }_{1} F_{1}\left(\frac{C}{A}, B ;-\frac{2 \nu A}{2 \nu+\delta}\right) .
$$

Substitution of (3) into the above yields

$$
P_{b}=\frac{B \lambda(\nu+\delta)}{B(2 \lambda+\mu)(\nu+\delta)-2 H C \nu \delta},
$$

where

$$
H=\frac{{ }_{1} F_{1}\left(\frac{C}{A}+1, B+1 ;-\frac{2 \nu A}{2 \nu+\delta}\right)}{{ }_{1} F_{1}\left(\frac{C}{A}, B ;-\frac{2 \nu A}{2 \nu+\delta}\right)} .
$$

Mean number of customers in the orbit. Let $N$ denote a generic random variable for the number of customers in the orbit at steady state. Then the mean $\mathbb{E} N$ is given by $\mathbb{E} N=p^{\prime}(1)+q^{\prime}(1)$. Therefore, according to Theorem 1 , we have

$$
\begin{aligned}
\mathbb{E} N= & g(0) e^{\frac{\lambda}{\delta}}\left\{\frac{2 \lambda+\mu+\delta}{\delta}{ }_{1} F_{1}\left(\frac{C}{A}, B ;-\frac{2 \nu A}{2 \nu+\delta}\right)\right. \\
& -\frac{C}{B} \frac{4 \lambda \nu+2 \nu \delta+2 \lambda \delta+\delta^{2}+\mu \nu+\mu \delta}{\lambda(\nu+\delta)} \\
& \times{ }_{1} F_{1}\left(\frac{C}{A}+1, B+1 ;-\frac{2 \nu A}{2 \nu+\delta}\right) \\
& \left.+\frac{C}{B(B+1)} \frac{2 \nu(3 \delta+\mu)}{\delta(2 \nu+\delta)}{ }_{1} F_{1}\left(\frac{C}{A}+2, B+2 ;-\frac{2 \nu A}{2 \nu+\delta}\right)\right\} .
\end{aligned}
$$

Substitution of (3) into the above yields

$$
\begin{aligned}
\mathbb{E} N= & \frac{B(\nu+\delta)}{B(2 \lambda+\mu)(\nu+\delta)-2 H C \nu \delta}\left\{\frac{\lambda(2 \lambda+\mu+\delta)}{\delta}\right. \\
& \left.-\frac{H C}{B} \frac{4 \nu \lambda+2 \nu \delta+2 \lambda \delta+\delta^{2}+\mu \nu+\mu \delta}{\nu+\delta}+\frac{K C \lambda}{B(B+1)} \frac{2 \nu(3 \delta+\mu)}{\delta(2 \nu+\delta)}\right\},
\end{aligned}
$$

where

$$
K=\frac{{ }_{1} F_{1}\left(\frac{C}{A}+2, B+2 ;-\frac{2 \nu A}{2 \nu+\delta}\right)}{{ }_{1} F_{1}\left(\frac{C}{A}, B ;-\frac{2 \nu A}{2 \nu+\delta}\right)} .
$$

\section{References}

[1] M. S. Aguir, O. Z. Akşin, F. Karaesmen, and Y. Dallery, On the interaction between retrials and sizing of call centers, European Journal of Operational Research 191 (2008), no. 2, 398-408.

[2] M. S. Aguir, F. Karaesmen, O. Z. Akşin, and F. Chauvet, The impact of retrials on call center performance, OR Spectrum 26 (2004), no. 3, 353-376. 
[3] J. R. Artalejo, A classified bibliography of research on retrial queues: progress in 19901999, Top 7 (1999), no. 2, 187-211.

[4] _ Accessible bibliography on retrial queues, Math. Comput. Model. 30 (1999), $1-6$.

[5] _ Accessible bibliography on retrial queues: Progress in 2000-2009, Math. Comput. Model. 51 (2010), 1071-1081.

[6] J. R. Artalejo and A. Gomez-Corral, Retrial Queueing Systems, Springer-Verlag, Berlin Heidelberg, 2008.

[7] J. R. Artalejo and A. Pla, On the impact of customer balking, impatience and retrials in telecommunication systems, Computers and Mathematics with Applications 57 (2009), no. 2, 217-229.

[8] A. Erdelyi, Higher Transcendental Functions, Vol. 1, McGraw-Hill, New York, 1953.

[9] G. I. Falin, A survey of retrial queues, Queueing Systems Theory Appl. 7 (1990), no. 2, $127-167$.

[10] G. I. Falin and J. G. C. Templeton, Retrial Queues, Chapman \& Hall, London, 1997.

[11] B. K. Kumar, G. Vijayalakshmi, A. Krishnamoorthy, and S. S. Basha, A single server feedback retrial queue with collisions, Computers \& Operations Research 37 (2010), no. 7, 1247-1255.

[12] T. Yang and J. G. C. Templeton, A survey on retrial queues, Queueing Systems: Theory and Applications 2 (1982), no. 3, 201-234.

Department of Mathematics Education

Chungbuk National University

Cheonguu 361-763, Korea

E-mail address: jeongsimkim@chungbuk.ac.kr 